\title{
Proton Charge Radius (PRad) Experiment at Jefferson Lab
}

\author{
C. Peng ${ }^{1, a}$ and H. Gao ${ }^{1,2, b}$ \\ ${ }^{1}$ Duke University, Durham, NC27708, U.S.A. \\ ${ }^{2}$ Duke Kunshan University, Kunshan, Jiangsu, China 215316
}

\begin{abstract}
The puzzle of proton charge radius was recently raised by the measurement of muonic hydrogen Lamb shift at Paul Scherrer Institute (PSI), whose results were seven standard deviations smaller than the CODATA recommended value. To investigate this discrepancy, the PRad experiment was proposed and approved at Thomas Jefferson National Accelerator Facility (JLab). The experiment will extract the proton charge radius with a sub-percent accuracy by measuring the cross-sections of unpolarized electronproton elastic scattering in an unprecedented low $Q^{2}$ region $\left(2 \times 10^{-4} \mathrm{GeV}^{2} / \mathrm{c}^{2}\right)$.
\end{abstract}

\section{Introduction}

The proton charge radius is an important quantity to understand the structure of proton. It is not only required for high precision tests of quantum electrodynamics (QED) calculations based on the hydrogen Lamb shift, but also crucial for understanding how quantum chromodynamics (QCD) works in the non-perturbative region. Recently, measurements of muonic hydrogen Lamb shift at PSI reported the most precise values of proton charge radius in 2010 [1] and 2013 [2], which were:

$$
\begin{aligned}
& r_{p}=0.84184 \pm 0.00067 \mathrm{fm} \mathrm{[1]} ; \\
& r_{p}=0.84087 \pm 0.00039 \mathrm{fm} \mathrm{[2]} .
\end{aligned}
$$

However, the PSI results were $7 \sigma$ away from the CODATA 2012 value [3], which is a compilation of the results from hydrogen Lamb shift measurements and elastic electron-proton $(e p)$ scattering experiments. As shown in Figure 1, a large discrepancy exists between the PSI values and the results from the recent $e p$ scattering experiments or data reanalyses, including the analysis of world $e p$ elastic scattering data by Sick [4], the result extracted from the elastic ep scattering measurements at Mainz Microtron by Bernauer et al. [5], the updated global fit of the form factors data by Zhan et al. [6], and the world data average suggested by Arrington [7].

This discrepancy is still not understood and is known as the "proton radius puzzle". It is of great interest to atomic, particle, and nuclear physicists. Many efforts have been made to understand the discrepancy: calculations of muonic hydrogen energy levels were refined, and were summarized in $[8,9]$; a muon-proton scattering measurement was approved at PSI [10] to search the possible difference between the muon and electron measurements. A high precision elastic ep scattering experiment

\footnotetext{
a e-mail: cp121@phy.duke.edu

be-mail: gao@phy.duke.edu
} 


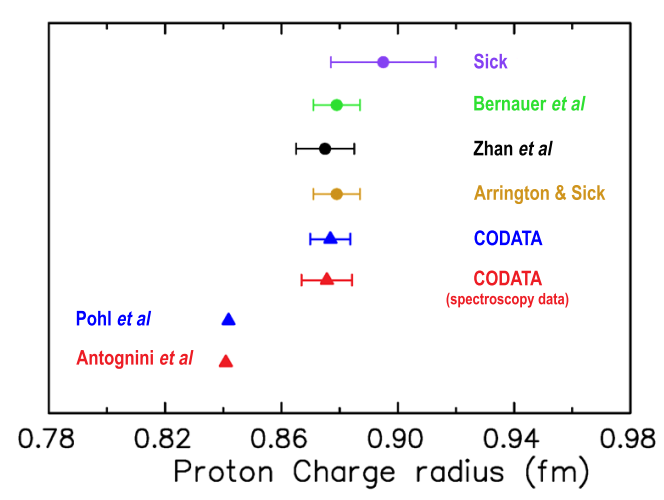

Figure 1. A compilation of values of the proton charge radius extracted from recent measurements and analysis.

with different systematical uncertainties is also necessary to evaluate the discrepancy, hence we have proposed the "high precision measurement of the proton charge radius" (PRad experiment) [11] in Hall B at Thomas Jefferson National Accelerator Facility (JLab). This report will introduce the experimental setup and its current status.

\section{Experimental Setup}

In 2012, the PRad experiment was approved at JLab with A rating and 15 days of 1.1 and $2.2 \mathrm{GeV}$ beam. As shown in Figure 2, this experiment utilizes a non-magnetic calorimetric setup. It will measure the cross-section of unpolarized elastic ep scattering at very low four momentum transfer square $\left(\mathrm{Q}^{2}\right)$, and extract the electric form factors from it.

The PRad target is a windowless hydrogen gas-flow target, which removes the typical background source of all previous ep experiments - the elastic or quasi-elastic scattering off the nuclei in the target windows. In addition, a five meters long, two-stages vacuum box connects the target chamber to the detectors, hence minimizes any possible background sources between these two subsystems.

The detector package includes a Hybrid Calorimeter (HyCal) and two Gas Electron Multiplier (GEM) chambers. The HyCal consists of $1152 \mathrm{PbWO}_{4}$ crystal modules as the central part and 576 lead glass modules as the outer part. The energy and position resolutions of the $\mathrm{PbWO}_{4}$ modules are $2.6 \% / \sqrt{E(\mathrm{GeV})}$ and $2.5 \mathrm{~mm} / \sqrt{E(\mathrm{GeV})}$, respectively, while the resolutions of the $\mathrm{Pb}$-glass modules are approximately doubled. Two GEM chambers will be combined and mounted on the HyCal box to cover the entire geometrical acceptance of HyCal. The GEM detector improves the position resolution to better than $0.1 \mathrm{~mm}$, and significantly reduces the systematical uncertainty in $Q^{2}$ determination. The detection angle range is $0.8^{\circ}$ to $8^{\circ}$, corresponding to a kinematic coverage of $Q^{2}=2 \times 10^{-4}-0.09 \mathrm{GeV}^{2} / \mathrm{c}^{2}$. The lowest $\mathrm{Q}^{2}$ region was never achieved before by magnetic spectrometer based measurements.

Scattered electrons from both Møller and ep elastic scattering will be simultaneously measured during the experiment. These two types of events within the kinematic coverage of this experiment can be easily separated by their energies. The cross sections of $e p$ elastic scattering thus can be nor- 


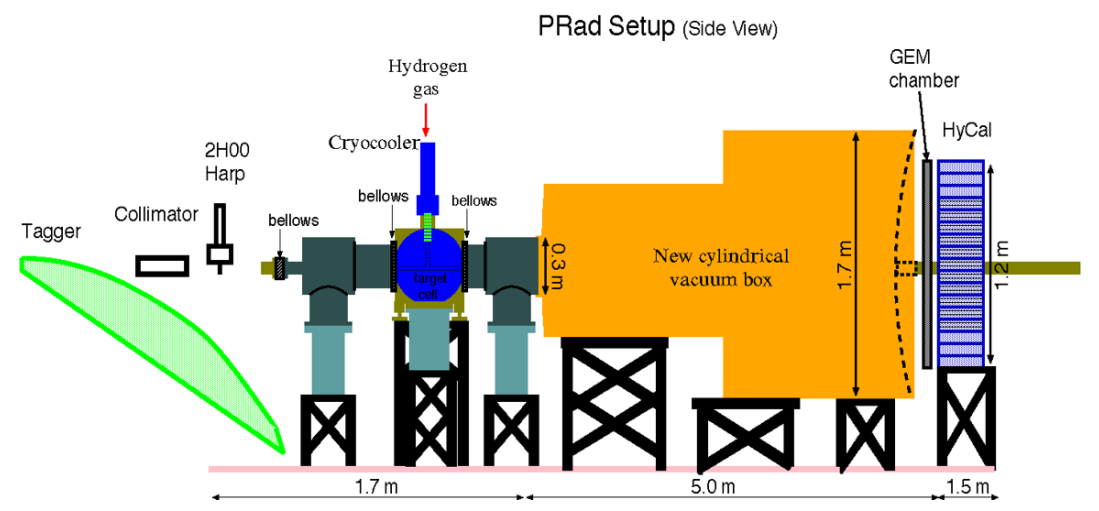

Figure 2. The proposed experimental layout (Not in scale). [11]

malized to those of the well known Møller process. This method significantly reduces the systematical uncertainty on determining the luminosity.

The experiment will eventually extract the electric form factor $\left(\mathrm{G}_{E}\right)$ from the measured crosssection data. According to the Rosenbluth formula,

$$
\sigma_{R}=\left(\frac{d \sigma}{d \Omega}\right)_{\text {exp }} /\left(\frac{\sigma_{M o t t}}{\epsilon} \frac{\tau}{1+\tau}\right)=\frac{\epsilon}{\tau} G_{E}^{p^{2}}+G_{M}^{p^{2}} .
$$

, where $\tau=Q^{2} /\left(4 M_{p}^{2}\right)$, and $\epsilon=\left(1+2(1+\tau) \tan ^{2} \frac{\theta}{2}\right)^{-1}$, the contribution from magnetic form factor $\left(G_{M}\right)$ strongly depends on $\mathrm{Q}^{2}$. Within the low $\mathrm{Q}^{2}$ region that is only achievable by $1.1 \mathrm{GeV}$ beam, the $G_{M}$ contribution is only $0.015 \%$ to $0.06 \%$, and hence negligible. At higher $\mathrm{Q}^{2}$, as the contribution of $\mathrm{G}_{M}$ becomes noticeable, we can either separate the two form factors using Rosenbluth method, or correct the $\mathrm{G}_{M}$ contribution by the existing world data.

\section{Simulation Results}

A full simulation program has been developed for the PRad experiment based on GEANT4 toolkit [12, 13]. The primary background source was found to be the electrons in the beam halo scattered off the nuclei in the target cell structures. To suppress the background, we enlarged the cell diameter, and planned to perform several empty target runs for background subtraction. The simulation including the empty target runs shows that the statistical uncertainties on the cross-sections will be less than $0.5 \%$ within the approved beam time, and the systematical uncertainty due to the subtraction can be less than $0.4 \%$.

In the simulation program, two independent event generators with radiative effects were used. One is based on the complete set of calculations in [14], which goes beyond the ultra-relativistic approximations (URA). The other one is ESEPP [15], whose first order bremsstrahlung is calculated without soft-photon approximation or URA. The study with these two event generators shows the systematical uncertainty due to the radiative corrections is less than $0.3 \%$.

The detector acceptance and $Q^{2}$ determination were also studied in the simulation. The implementation of GEM significantly improves the position resolution and hence the determination of $Q^{2}$, 
which helps control the systematical uncertainties of the acceptance. Reconstruction of tracking between the HyCal and GEM can further reduce the background from the target cell structures. Based on this simulation and the previous behavior of $\mathrm{HyCal}$ in the PrimEx experiment [16], a total uncertainty of $0.4 \%$ regarding the detector acceptance was assumed.

In addition, we have studied the rejection of neutral particles and the contamination of pions. Both uncertainties are less than $0.1 \%$. A full simulation including all the above factors was performed. The proton charge radius extracted by a dipole fitting from simulation data indicates a total uncertainty of less than $0.7 \%$.

\section{Summary}

The large experimental discrepancy on one of the fundamental quantities, the proton charge radius, has puzzled the communities since 2010. The PRad experiment aims to investigate this puzzle by extracting the proton charge radius with a sub-percent precision from the elastic electron-proton scattering measurement. Its magnetic-free calorimetric setup will reach the unprecedented low $\mathrm{Q}^{2}$ region, and also significantly improve the systematical uncertainties that were typical in previous magnet spectrometer experiments. With these advantages, results from PRad experiment will directly impact on the "proton radius puzzle".

\section{Acknowledgements}

We thank members of the entire PRad collaboration. This project is supported in part by U.S. Department of Energy under contract number DE-FG02-03ER41231 and NSF MRI award PHY-1229153.

\section{References}

[1] R. Pohl et al., Nature 466, 213 (2010).

[2] A. Antognini et al., Science 339, 417 (2013).

[3] P.J. Mohr, B.N. Taylor, and D.B. Newell, Rev. Mod. Phys. 84, 1527 (2012).

[4] I. Sick, Phys. Lett. B 576, 62 (2003).

[5] J.C. Bernauer et al., Phys. Rev. Lett. 105, 242001 (2010).

[6] X. Zhan et al., Phys. Lett. B 705, 59 (2011).

[7] J. Arrington and I. Sick, arXiv:1505.02680v1 [nucl-ex] (2015).

[8] U.D. Jentschura, Ann. Phys. (N.Y.) 326, 500 (2011).

[9] A. Antognini et al., arXiv:1208:2637v2 [atom-ph] (2012).

[10] R. Gilman et al., arXiv:1303:2160v1 [nucl-ex] (2013).

[11] A. Gasparian et al., JLab experiment proposal E12-11-106 (2011). Spokespersons: D. Dutta, H. Gao, A. Gasparian (contact), M. Khandaker; A. Gasparian et al., update to proposal C12-11-106 (2012).

[12] S. Agostinelli et al., NIM A 506, 3 (2003).

[13] J. Allison et al., IEEE Trans. Nucl. Sci 53, 1 (2006).

[14] I. Akushevich, H. Gao, A. Ilyichev, and M. Meziane Eur. Phys. J. A 51, 1 (2015).

[15] A. V. Gramolin et al., J. Phys. G. 41, 115001 (2014).

[16] M. Kubantsev et al., AIP Conf. Proc. 867, 51 (2006). 